\title{
A Mollifier Useful for Approximations in Sobolev Spaces and Some Applications to Approximating Solutions of Differential Equations*
}

\author{
By Stephen Hilbert
}

\begin{abstract}
For a given uniform grid of $E^{N}$ ( $N$-dimensional Euclidean space) with mesh $h$, a class of smoothing functions (mollifiers) is constructed. If a function is an element of the Sobolev space $H_{2}^{m}$, then the error made by replacing the given function by a smoother $\left(C^{\infty}\right)$ function (which is the given function convolved with one of the mollifiers) is bounded by a constant times $h^{m}$.

This result is used to construct approximations for functions using Hermite or spline interpolation, even though the function to be approximated need not satisfy the continuity conditions necessary for the existence of a Hermite or spline interpolate. These techniques are used to find approximations to the generalized solution of a second order elliptic Neumann problem.
\end{abstract}

I. Introduction. One of the most useful applications of approximation theory is in approximating the solutions of differential equations. Many approximation schemes demand that the function which is being approximated satisfy certain continuity conditions (e.g. Hermite and spline interpolation). Unfortunately, in many cases the solution to a given differential equation may not satisfy these conditions, or the theoretical solution is an element of Sobolev space and may not satisfy the necessary continuity conditions for such schemes.

One procedure for dealing with this type of problem is to "smooth" the function by convolving the given function with a second function (called a mollifier). Then, one approximates the smoothed function which does possess the desired continuity properties. However, another source of error has been introduced, the error made by replacing the original function by the "smoothed" function. This leads to the problem of constructing mollifiers which have the property that the "smoothed" function is "close" to the original function.

In this note, we shall demonstrate a method for constructing a class of mollifiers with the property that if a function $u$ is in the Sobolev space $H_{2}^{m}$, then the error made by replacing $u$ by $u$, convolved with one of the mollifiers, is $m$ th order. This will enable us to construct approximants which are $m$ th order accurate for functions in $H^{m}$ by using interpolation techniques (even if the original function could not be interpolated).

Using Hermite and spline interpolation techniques, we will construct two sub-

Received January 18, 1972.

AMS (MOS) subject classifications (1970). Primary 65N30, 41A25; Secondary 41A15, $46 \mathrm{E} 35$.

Key words and phrases. Finite element method, Galerkin method, Rayleigh-Ritz method, Hermite interpolation, spline, mollifier, approximation, Sobolev space, boundary value problem.

* This paper contains part of the author's doctoral dissertation which was directed by Professor James H. Bramble of Cornell University and submitted to the University of Maryland. 
spaces of approximations which are $m$ th order accurate for functions in $H^{m}$. Such subspaces are of crucial importance in the finite element method, Galerkin method and the least squares method of Bramble and Schatz (see [4], [7]).

Finally, we use these subspaces to get error estimates for the approximate solution of a second order elliptic problem.

II. Preliminaries and Conventions. Let $R$ be a bounded domain in Euclidean $N$ space, $E^{N}$. We will assume $R$ satisfies a restricted cone condition, that is, there is a finite open cover of $\partial R$, the boundary of $R,\left\{\mathcal{O}_{i}\right\}$ and cones $C_{i}$ with vertices at the origin such that for all $x \in \mathcal{O}_{i} \cap R, x+C_{i}$ is contained in $R$.

We shall consider complex valued functions defined on $R$. As usual, we denote by $L_{2}(R)$ the completion of the functions $f$ defined on $R$ such that $\int_{R}|f(x)|^{2} d x=$ $\|f\|_{R}^{2}$ exists and is finite. $\alpha, \beta, \gamma$, and $\tau$ will denote multi-indices $\alpha=\left(\alpha_{1}, \cdots, \alpha_{N}\right)$ with $|\alpha|=\sum_{i=1}^{N} \alpha_{i}$ and $D^{\alpha}=\left(\partial / i \partial x_{1}\right)^{\alpha_{1}} \ldots\left(\partial / i \partial x_{N}\right)^{\alpha_{N}}$. For any nonnegative integer $m, H^{m}(R)$ is the set of all distributions $u$ such that the distributional derivatives $D^{\alpha} u$ are in $L_{2}(R)$ for all $|\alpha| \leqq m$. The norm on $H^{m}(R)$ is given by $\|u\|_{m, R}^{2}=\sum_{|\alpha| \leqq m}\left\|D^{\alpha} u\right\|_{R}^{2}$.

We will also consider the space of functions which have continuous derivatives of order up to and including $m$ in $R$. We denote this space by $C^{m}(R)$.

If a function $u$ is infinitely differentiable in $R$ and vanishes identically outside of some compact set contained in $R$, then we say $u$ is in $C_{0}^{\infty}(R)$.

Consider $C_{0}^{\infty}\left(E^{N}\right)$ which we will always denote as simply $C_{0}^{\infty}$. The completion of $C_{0}^{\infty}$ under the norm $\|u\|^{2}=\int_{E^{N}}|u(x)|^{2} d x$ is $L_{2}$. We define $H^{m}$ for any positive integer $m$ as the completion of $C_{0}^{\infty}$ under the norm $\|u\|_{m}^{2}=\sum_{|\alpha| \leqq m}\left\|D^{\alpha} u\right\|^{2}$.

Now let $\rho$ be the diameter of the domain $R$. Let $K$ be any subset of the set of multi-indices $\gamma$ of length $m$ (i.e., $|\gamma|=m$ ) which contains the indices $\gamma$ with $\gamma_{k}=$ $m, \gamma_{i}=0$ for $j \neq k$ and $k=1, \cdots, N$. The set of polynomials $q$ such that $D^{r} q=0$ for all $\tau \in K$ will be denoted by $P_{K}$. The following is Theorem 2 of [3].

THEOREM 2.1. Let $F$ be a linear functional on $H^{m}(R)$ satisfying

(a) $|F(u)| \leqq C \sum_{|\alpha| \leqq m} \rho^{|\alpha|-N / 2}\left\|D^{\alpha} u\right\|_{R}$ where $C$ is independent of $\rho$ and $u$ and (b) $F(q)=0$ for $q \in P_{K}$.

Then, there is a constant $C_{1}$ independent of $\rho$ and $u$ such that $|F(u)| \leqq C_{1} \rho^{m-N / 2}$ $\cdot \sum_{\tau \in K}\left\|D^{r} u\right\|_{R}$.

We will always assume that all functions are restricted to the domain $R$. Hence, we can regard $P_{K}$ as a subspace of $H^{m}(R)$.

In this paper, $C$ will be used to denote a generic constant not necessarily the same in any two places. Also, all norms which appear in this paper are $L_{2}$ or $H_{2}^{m}$ norms.

III. A Special Mollifier. If a function $f$ satisfies certain continuity properties, then there are certain subspaces $S$ such that an approximation to $f, f_{1}$, is in $S$. For example, to find an interpolate of $f, f$ must usually be continuous. Now, if $f$ does not have the necessary continuity properties, it still may be possible to find an approximation of $f$ in $S$. The method we will use to find the approximant is first to smooth $f$ so that it does satisfy the continuity properties and then to approximate the smoothed function. If the difference between $f$ and the smoothed function is of the same accuracy as the original approximation, then the method will give an accurate approximation to $f$ in $S$, even when $f$ is not continuous. We will now make these ideas precise. 
We will need the Calderón extension theorem. Let $R$ be a bounded domain satisfying the restricted cone property. Then, there exists a bounded linear transformation $P$ of $H^{m}(R)$ into $H^{m}$ such that for any $u \in H^{m}(R), P u(x)=u(x)$ for all $x$ in $R$. The proof may be found in Agmon [1, p. 171]. Hence, there is a constant $C$ independent of $u$ such that $\|P u\|_{m} \leqq C\|u\|_{m, R}$.

For two square integrable functions $f$ and $g$, the convolution of $f$ with $g$, denoted by $f * g$, is defined as

$$
f * g(x)=\int_{E^{N}} f(x-y) g(y) d y=g * f(x) .
$$

For a function $\phi$ in $C_{0}^{\infty}$, we can define a linear operator $T$ on locally integrable functions $f$ by $T f(x)=\phi * f(x)$. Since $D(T f)=D \phi * f$, if $\phi$ is infinitely differentiable, then $T f$ is. Hence, $\phi$ is called a mollifier or smoothing function. In this chapter, we will construct a mollifier with the property $T f=f$ for $f$ in certain classes of polynomials.

Consider the function $\phi_{\epsilon}$ in $C_{0}^{\infty}\left(E^{1}\right)$ given by

$$
\begin{aligned}
\phi_{\epsilon}(x) & =\frac{\rho}{\epsilon} \exp \left[-\frac{\epsilon^{2}}{\epsilon^{2}-x^{2}}\right] & & \text { for } x \in(-\epsilon, \epsilon), \\
& =0 & & \text { if }|x|>\epsilon,
\end{aligned}
$$

where $1 / \rho=\int_{E^{2}} \phi_{1}(x) d x$. By a change of variable, it is easy to see that $\int \phi_{\epsilon}(x) d x=1$ for any $\epsilon>0$. Hence, $\phi_{\epsilon} * c=c$ for any constant $c$. Consider $\left(\phi_{\epsilon} * x\right)(y)-y$ as a function of $y$. Since its derivative is zero, $\left(\phi_{\epsilon} * x\right)(y)-y$ is constant. Now, $x$ is an odd function and $\phi_{\epsilon}$ is an even function, thus, when $y=0$, the constant vanishes. Thus, the operator associated with $\phi_{\epsilon}$ reproduces polynomials of degree $\leqq 1$. It follows that, for any real number $r$, the operator associated with $r \phi_{\epsilon}+(1-r) \phi_{\epsilon / 2}$ reproduces polynomials of degree $\leqq 1$. We denote $r \phi_{\epsilon}+(1-r) \phi_{\epsilon / 2}$ by $\phi_{\epsilon, r}^{(2)}$. Consider $\left(\phi_{\epsilon, r}^{(2)} * x^{2}\right)(y)-y$; this is a constant since its derivative is zero. Hence, if we choose $\bar{r}$ so that $\left(\phi_{\epsilon, \bar{r}}^{(2)} * x^{2}\right)(0)=0$, the operator associated with $\phi_{\epsilon, \bar{r}}^{(2)}$ will reproduce polynomials of degree $\leqq 2$. By a change of variables, $\left(\phi_{\epsilon / 2} * x^{2 m}\right)(0)=$ $2^{-2 m}\left(\phi_{\epsilon} * x^{2 m}\right)(0)$. Hence, $\left(\phi_{\epsilon, r}^{(2)} * x^{2}\right)(0)=\left(\phi_{\epsilon} * x^{2}\right)(0)[r+(1-r) 1 / 4]$, so $\bar{r}=-1 / 3$. We denote $\phi_{\epsilon,-1 / 3}^{(2)}$ as simply $\phi_{\epsilon}^{(2)}$. Since $\phi_{\epsilon}^{(2)}$ is even and $x$ is odd, the operator associated with $\phi_{\epsilon}^{(2)}$ reproduces polynomials of degree $\leqq 3$. Letting $\phi_{\epsilon, r}^{(3)}=r \phi_{\epsilon}^{(2)}+$ $(1-r) \phi_{\epsilon / 2}^{(2)}$ and following the above procedure, we can construct $\phi_{\epsilon}^{(3)}$ and the operator associated with $\phi_{\epsilon}^{(3)}$ will reproduce polynomials of degree $\leqq 5$. Hence, we can construct a function $\phi_{\epsilon}^{(m)}$ which is $C_{0}^{\infty}$, and the operator associated with $\phi_{\epsilon}^{(m)}$ will reproduce polynomials of degree $\leqq 2 m-1$. We remark that $\int \phi_{\epsilon}^{(n)}(x) d x=1$ for any $\epsilon>0$ and any nonnegative integer.

We shall define $\phi_{\epsilon}^{(m)}(x)$ as $\prod_{j=1}^{N} \phi_{\epsilon i}^{(m)}\left(x_{i}\right)$, where $x=\left(x_{1}, \cdots, x_{N}\right)$ and $\epsilon=$ $\left(\epsilon_{1}, \cdots, \epsilon_{N}\right)$. It is obvious (by Fubini's Theorem) that the operator associated with $\phi_{\epsilon}^{(m)}$ will reproduce polynomials of degree at most $2 m-1$ in each variable.

Next, let $R_{i}$ be hypercubes with sides of length $h$ that satisfy $E^{N}=\bigcup_{i} R_{i}$ and $R_{i} \cap R_{i}=\varnothing$ or an edge of $R_{i}$ for $i \neq j$. For any $x$ in $E^{N}$, define $S_{h}(x)$ as the set of all hypercubes $R_{i}$ such that there is an element $y$ in $R_{i}$ satisfying $\left|x_{i}-y_{i}\right| \leqq h$ for all $j=1, \cdots, N$. Note that there is an upper bound which is independent of $x$ for the number of hypercubes in $S_{h}(x)$.

We will need the following Lemma. 
LEMMA. Let $u$ be an element of $L_{2}$. Then, for any positive integer $m$, there are positive constants $C$ and $C_{1}$ which are both independent of $u$ and $h$ such that

$$
\left|\phi_{h}^{(m)}(y)\right| \leqq C h^{-N} \text { for any } y \text { in } E^{N}
$$

and

$$
\left\|\phi_{h}^{(m)} * u\right\|_{S_{h}(x)} \leqq C_{1} h^{-N / 2}\|u\|_{\mathrm{Ch}(\mathrm{x})} \text { for any } x \text { in } E^{N},
$$

where $\mathrm{C}_{\mathrm{h}}(\mathrm{x})$ is simply the set of points $z$ in $E^{N}$ such that $z$ is in $S_{h}(w)$ for some $w$ in $S_{h}(x)$.

Proof. Let $y_{i}=h z_{i}$, then $\phi_{h}\left(y_{i}\right)=(1 / h) \phi_{1}\left(z_{i}\right)$. Since $\phi_{h / 2^{n}}\left(y_{i}\right)=\left(2^{n} / h\right) \phi_{1}\left(2^{n} z_{i}\right)$ and $\phi_{h}^{(m)}$ is a linear combination of $\phi_{h / 2^{k}}$ for $k=0,1, \cdots, m-1$, we can express $\phi_{h}^{(m)}$ as a combination of $(1 / h) \phi_{1}$ evaluated at different points. Since $\phi_{1}$ is bounded independently of $h$ and $y$, the first inequality of the lemma is proved.

The second inequality follows by expressing $\phi_{h}^{(m)} * u$ as an integral, interchanging the order of integration, using the first inequality and the fact that $\phi_{h}^{(m)}$ has support which is proportional to $h^{N}$.

We will now prove the main result of this section.

THEOREM 3.1. Suppose $u$ is a function which is in $H^{m}$. Then, there is a constant $C$ independent of $u$ and $h$ such that

$$
\left\|D^{\alpha}\left(\phi_{h}^{(k)} * u-u\right)\right\| \leqq C h^{m-|\alpha|} \sum_{j=1}^{N}\left\|D_{i}^{(m)} u\right\|
$$

for any index $\alpha$ with $|\alpha| \leqq m$ and $k$ is greater than or equal to the greatest integer contained in $\frac{1}{2}(m+1) . D_{i}^{(m)} u$ is the mth partial derivative of $u$ in the jth coordinate direction.

Proof. Let $u$ be in $H^{m}$. Then choose $x$ in $E^{N}$ and $\psi$ in $C_{0}^{\infty}$. Define a functional by

$$
F(u, \psi, x)=\int_{S_{h}(x)}\left[\left(\phi_{h}^{(k)} * u\right)(y)-u(y)\right] \bar{\psi}(y) d y .
$$

It is easy to see that $F$ is linear in $u$ and that $F(u, \psi, x)$ is defined for any $u$ in $L_{2}$. Also, by the construction of $\phi_{h}^{(k)}$, we have $F(p, \psi, x)=0$ for any polynomial $p$ of degree at most $m-1$ in each variable. Using the Schwartz inequality, we can obtain

$$
|F(u, \psi, x)| \leqq\left\|\phi_{h}^{(k)} * u-u\right\|_{S_{h}(x)}\|\psi\|_{S_{h}(x)} \leqq C h^{-N / 2}\|u\|_{\mathrm{C}_{\mathrm{h}}(\mathrm{x})}\|\psi\|_{S_{\mathrm{h}}(x)},
$$

where $C$ is independent of $u$ and $h$ by our lemma. So by the theorem in Section 2, we have

$$
|F(u, \psi, x)| \leqq C h^{m-N / 2} \sum_{j=1}^{N}\left\|D_{i}^{(m)}(u)\right\|_{\mathrm{Ch}_{\mathrm{h}}(\mathrm{x})}\|\psi\|_{S_{\mathrm{h}(x)}} .
$$

Now integrate $|F(u, \psi, x)|^{2}$ with respect to $x$ over any hypercube $R_{i}$ to obtain

$$
\left(\int_{R_{i}}|F(u, \psi, x)|^{2} d x\right)^{1 / 2} \leqq C h^{m} \sum_{i=1}^{N}\left\|D_{i}^{(m)} u\right\|_{\mathrm{C}_{\mathrm{i}}}\|\psi\|_{\mathrm{C}_{\mathrm{i}}}
$$

where $\mathrm{C}_{\mathrm{i}}$ is the set of all points $y$ such that $S_{h}(y) \cap R_{i} \neq \varnothing$. Since the $L_{2}$ norm of any function $f$ in $R_{i}$ is the supremum of $\left|\int_{R_{i}} f \psi\right|$ for $\psi$ with norm 1 in $L_{2}\left(R_{i}\right)$ and $C^{\infty}\left(R_{i}\right)$ is dense in $L_{2}\left(R_{i}\right)$, we obtain 


$$
\left\|\phi_{h}^{(k)} * u-u\right\|_{R_{i}} \leqq C h^{m} \sum_{j=1}^{N}\left\|D_{i}^{(m)} u\right\|_{\mathrm{C}_{\mathrm{i}}} .
$$

Summing over all possible indices $i$ and, since each $C_{\mathrm{i}}$ is within a combination of a finite number of hypercubes $R_{i}$, we obtain the theorem in the case $\alpha=(0, \cdots, 0)$. The theorem follows by considering $h^{|\alpha|} F\left(D^{\alpha} u, \psi, x\right)$ for all other multi-indices $\alpha$ with $|\alpha| \leqq m$.

Now, if $R$ is a bounded domain in $E^{N}$, for $u$ in $H^{m}(R), P u$ (the Calderón extension of $u$ ) is in $H^{m}$, so by the previous theorem

$$
\left\|D^{\alpha}\left(\phi_{h}^{(k)} * P u\right)-P u\right\| \leqq C h^{m-|\alpha|} \sum_{j=1}^{N}\left\|D_{i}^{(m)} P u\right\| .
$$

Now, since $P u(x)=u(x)$ for any $x$ in $R$ and $C^{\infty}(R) \cap H^{m}(R)$ is dense in $H^{m}(R)$. we have $D^{\alpha} P u(x)=D^{\alpha} u(x)$ for all indices $\alpha$ such that $|\alpha| \leqq m$ and for any $x$ in $R$, Thus, we have proved the following corollary.

Corollary. Let $u$ be in $H^{m}(R)$. Then there is a constant $C$ independent of $u$ and $h$ such that

$$
\left\|D^{\alpha}\left[\left(\phi_{h}^{(k)} * P u\right)-u\right]\right\|_{R} \leqq C h^{m-|\alpha|} \sum_{i=1}^{N}\left\|D_{i}^{(m)} u\right\|_{R}
$$

for any index $\alpha$ such that $|\alpha| \leqq m$ and for any $k$ larger than or equal to the greatest integer contained in $\frac{1}{2}(m+1)$.

IV. Applications to Approximation Theory. In this section, we will prove a general theorem on approximation, and then apply the theorem to the special cases of Hermite and spline interpolation.

THEOREM 4.1. Let $h$ be a positive parameter. Assume there is a subspace $S_{m, h}(R)$ of $H^{m}(R)$ such that, for any function $u$ in $C^{\infty}(R) \cap H^{m}(R)$, there is an element $v$ of $S_{m, h}(R)$ satisfying $\left\|D^{\alpha}(u-v)\right\|_{R} \leqq C h^{m-|\alpha|}\|u\|_{m, R}$ where $C$ is independent of $h$ and $u$ and $\alpha$ is any index such that $|\alpha| \leqq m$. Then, for any $u$ in $H^{m}(R)$, there is $a w$ in $S_{m, h}(R)$ such that $\left\|D^{\alpha}(u-w)\right\|_{R} \leqq C h^{m-|\alpha|}\|u\|_{m, R}$ for any index $\alpha$ such that $|\alpha| \leqq m$ and $C$ is independent of $u$ and $h$. The result also holds for $R=E^{N}$.

Proof. Let $u$ be in $H^{m}(R)$, then by the theorem of Section 3, there is a function $u_{h}=\phi_{h}^{(k)} * P u$ (for $\left.k \geqq\left[\frac{1}{2}(m+1)\right]\right)$ and $\left\|D^{\alpha}\left(u-u_{h}\right)\right\|_{R} \leqq C h^{m-|\alpha|}\|u\|_{m, R}$.

Since $\phi_{h}^{(k)}$ is in $C^{\infty}, u_{h}$ is in $C^{\infty}(R) \cap H^{m}(R)$, so by assumption, there exists an element $w$ of $S_{m, h}(R)$ such that $\left\|D^{\alpha}\left(u_{h}-w\right)\right\|_{R} \leqq C h^{m-|\alpha|}|| u_{h} \|_{m, R}$. Using the Schwartz inequality on the functions $P u\left[\phi_{h}^{(k)}\right]^{1 / 2}$ and $\left[\phi_{h}^{(k)}\right]^{1 / 2}$, one easily obtains

$$
\left|u_{h}(x)\right| \leqq\left(\int_{E^{N}}[P u(y)]^{2}\left[\phi_{h}^{(k)}(x-y)\right] d y\right)^{1 / 2} .
$$

By Fubini's theorem, we can now obtain $\left\|u_{h}\right\|_{R} \leqq C\|P u\|$, where $C$ is independent of $h$ and $u$. Using the same technique on $D^{\alpha} u_{h}$ for all indices $\alpha$ such that $|\alpha| \leqq m$, we have $\left\|u_{h}\right\|_{m, R} \leqq C\|P u\|_{m} \leqq C_{1}\|u\|_{m, R}$, where the constants are independent of $h$ and $u$. The proof is completed by using the triangle inequality on $u-w=u-u_{h}+$ $u_{h}-w$.

This theorem proves that any subspace of approximations of the continuous elements of a Sobolev space is in fact a subspace of approximations of every element 
of the Sobolev space. Thus, interpolation techniques can be used even when there are discontinuous elements in the Sobolev space.

Consider the case when $u$ is in $H^{2 m}(R)$. If $u$ is in $C^{2 m-1}(R)$, then the $m$ th Hermite interpolate of $u, u_{m}$, exists and $\left\|D^{\alpha}\left(u-u_{m}\right)\right\|_{R} \leqq C h^{2 m-|\alpha|} \sum_{\tau \in K}\left\|D^{\tau} u\right\|_{R}$, where $K$ is a (proper) subset of the indices of length $2 m$. This was proved in [3].

Now, if $u$ is in $H^{2 m}(R)$, form $u_{h}=\phi_{h}^{(m)} * P u$; since $u_{h}$ will be an element of $C^{\infty}(R) \cap$ $H^{2 m}(R)$, the $m$ th Hermite interpolate of $u_{h}$, denoted $u_{h, m}$, exists. In fact, one can show, using the lemma of Section 3 and the techniques of [3], that

$$
\left\|D^{\alpha}\left(u_{h}-u_{h, m}\right)\right\|_{R} \leqq C h^{2 m-|\alpha|} \sum_{\tau \in K}\left\|D^{\tau} u\right\|_{R}
$$

for all indices $\alpha$ with $|\alpha| \leqq 2 m$ and $0 \leqq \alpha_{i} \leqq m-1$.

Now, define $H^{(m)}$ as the subspace of $C^{m-1}\left(E^{N}\right)$ such that $u$ is in $H^{(m)}$ if and only if $u$ is a polynomial of degree $\leqq 2 m-1$ in each variable in each $R_{i}$ and $u$ is in $C^{m-1}\left(E^{N}\right) . H^{(m)}(R)$ is the set of restrictions to $R$ of the elements of $H^{(m)}$.

THEOREM 4.3. Let $u$ be in $H^{2 m}(R)$. Then there is an element $v$ of $H^{(m)}(R)$ such that $\left\|D^{\alpha}(u-v)\right\|_{R} \leqq C h^{2 m-|\alpha|} \sum_{\tau \in K}\left\|D^{r} u\right\|_{R}$ where $C$ is independent of $h$ and $u$ for any index $\alpha$ with $|\alpha| \leqq 2 m$ and $0 \leqq \alpha_{i} \leqq m-1$ for $i=1, \cdots, N$. The result holds for $R=E^{N}$.

Proof. We note that the set $K$ always contains indices $\gamma$ of the form $\gamma_{i}=2 m, \gamma_{i}=$ 0 for $i \neq j, i, j=1, \cdots, N$. Hence,

$$
\sum_{\tau \in K}\left\|D^{\tau} u\right\|_{R} \geqq \sum_{j=1}^{N}\left\|D_{j}^{(2 m)} u\right\|_{R} .
$$

Thus, by Theorem 3.1 and (4.2), the theorem follows.

We remark that we could have partitioned $E^{N}$ into rectangles instead of hypercubes. The same type of analysis would give local error bounds in terms of the longest side of the rectangle in each rectangle. Thus, if the ratio of the longest side to the shortest side of each rectangle is bounded above and below independently of the rectangle and if the longest side of each rectangle is bounded above independently of the rectangle, we would obtain error bounds with the upper bound of the longest sides replacing $h$.

In [2], it was shown that if $u$ is in $H^{k}$ with $k>N / 2$, then there is a spline interpolant of order $k$ for $u, S_{k}(x, u)$ and $\left\|S_{k}-u\right\|_{i} \leqq C h^{k-i}\|u\|_{k}$ for $0 \leqq j \leqq k$. So we obtain the following results.

THEOREM 4.4. Let $u$ be in $H^{k}$. Then there exists a spline $w$ of order $k$ such that $\|u-w\|_{i} \leqq C h^{k-i}\|u\|_{k}$ for $0 \leqq j \leqq k$ with $C$ independent of $h$ and $u$.

Proof. Form $u_{h}=\phi_{h}^{(s)} * u$, where $s$ is the greatest integer contained in $\frac{1}{2}(k+1)$. Then, by Theorem 3.1, $\left\|u-u_{h}\right\|_{i} \leqq C h^{k-i}\|u\|_{k}$ for $0 \leqq j \leqq k$. So, by Theorem 4.1, we have just shown that $S_{k}\left(x, u_{h}\right)$ is a spline of order $k$ such that $\left\|S_{k}\left(\cdot, u_{h}\right)-u_{h}\right\|_{i} \leqq$ $C h^{k-i}\|u\|_{k}$ for $0 \leqq j \leqq k$.

Hence, let $w(x)=S_{k}\left(x, u_{h}\right)$ and the theorem follows.

COROLlaRY. Let $u$ be in $H^{k}(R)$. Let $S$ be the set of restrictions to $R$ of splines of order $k$. Then there is a $v \in S$ such that $\|u-v\|_{i, R} \leqq C h^{k-i}\|u\|_{k, R}$ for $0 \leqq j \leqq k$ with $C$ independent of $h$ and $u$.

Proof. Let $P u$ be the Calderón extension of $u$. Hence, $P u$ is in $H^{k}$. By Theorem 4.1, there is a spline of order $k$ such that $\|P u-w\|_{i} \leqq C h^{h-i}\|P u\|_{k}$ for $0 \leqq j \leqq k$. 
Thus, $\|u-w\|_{j, R} \leqq\|P u-w\|_{i} \leqq C h^{k-i}\|P u\|_{k} \leqq C h^{k-i}\|u\|_{k, R}$ by the continuity of the Calderón extension for $0 \leqq j \leqq k$. Hence, $w$ restricted to $R$ is in $S$ and the corollary follows with $v$ taken to be the restriction of $w$ to $R$.

Thus, we have exhibited two examples of subspaces $S_{m, h}(R)$. Notice that the spaces were formed by interpolation techniques which demand continuity, but by using the mollifiers of Section 3, we could extend the results to all elements of the Sobolev space.

V. A Problem with Natural Boundary Conditions. We will consider an operator

$$
L u(x)=-\sum_{i, j=1}^{N} \frac{\partial}{\partial x_{i}}\left(a_{i j}(x) \frac{\partial}{\partial x_{i}} u(x)\right)+c(x) u(x),
$$

where $a_{i j}(x)$ and $c(x)$ are real-valued functions with $c(x)>k>0$ for all $x \in R$, $a_{i j}(x)=a_{i i}(x)$ for all $x \in R$ and $\sum_{i, i=1}^{N} a_{i j}(x) \xi_{i} \xi_{i}>k_{1}|\xi|^{2}$ for any $x \in R$ and for any $\xi$ an $N$ vector of real numbers with $|\xi|^{2}=\xi_{1}^{2}+\cdots+\xi_{N}^{2}$. We will assume that $a_{i j}$ and $c$ are in $C^{1}(R)$ where $R$ is a bounded domain in $E^{N}$.

Consider the problem of finding a generalized solution of

$$
\begin{gathered}
L u(x)=f(x) \text { for } x \text { in } R, f \in L_{2}(R), \\
\sum_{i, j=1}^{N} a_{i j}(x) \frac{\partial u(x)}{\partial x_{j}} \cos \left(\nu, x_{i}\right)=0 \quad \text { for } x \in \partial R,
\end{gathered}
$$

where $\nu$ is the outer normal on $\partial R$. We define a bilinear form on $H^{1}(R) \times H^{1}(R)$ by

$$
a(u, v)=\int_{R}\left[\sum_{i, j=1}^{N} a_{i j}(x) \frac{\partial u}{\partial x_{i}}(x) \frac{\overline{\partial v}}{\partial x_{i}}(x)+c(x) u(x) \bar{v}(x)\right] d x .
$$

Now, by our assumptions, $|a(u, u)| \geqq \min \left(k, k_{1}\right)\|u\|_{1, R}^{2}$.

By a generalized solution of (*), we mean a function $u$ in $H^{1}(R)$ such that

$$
a(u, v)=\int_{R} f(x) \bar{v}(x) d x \text { for all } v \text { in } H^{1}(R) .
$$

Now, we will approximate $u$ by a solution of a Rayleigh-Ritz-Galerkin problem. The Rayleigh-Ritz-Galerkin problem is to find a function $\phi$ in $S$ such that $a(\phi, w)=$ $\int_{R} f(x) \bar{w}(x) d x$ for all $w$ in $S$ where $S$ is a finite-dimensional subspace of $H^{1}(R)$. This problem has a unique solution $\phi$ in $S$ if and only if the problem $\psi \in S, a(\psi, w)=0$ for all $w$ in $S$ has $\psi \equiv 0$ as its unique solution. Since $\psi$ is in $S$, we have $a(\psi, \psi)=0$, but this means $\|\psi\|_{1, R}=0$. Hence, the Rayleigh-Ritz-Galerkin problem always has a unique solution $\phi$ which is in the subspace $S$.

Hence, $a(u-\phi, w)=0$ for all $w \in S$. Thus, $a(u-\phi, \phi-w)=0$ for all $w \in S$ since $\phi$ is in $S$. Now, $a(u, u) \geqq K_{2}\|u\|_{1, R}^{2}$ where $K_{2}=\min \left(k, k_{1}\right)$. Thus, by the Schwartz inequality,

$$
\begin{aligned}
\|u-\phi\|_{1, R}\|u-w\|_{1, R} & \geqq C|a(u-\phi, u-w)|=C|a(u-\phi, u-\phi)| \\
& \geqq C K_{2}\|u-\phi\|_{1, R}^{2} .
\end{aligned}
$$

Hence,

$$
\|u-\phi\|_{1, R} \leqq C K_{2} \inf _{w \in S}\|u-w\|_{1, R} .
$$


Now, if the solution $u$ is in $H^{2 l}(R)$, choose $S$ as $H^{(l)}(R)$ (the restriction to $R$ of $l$ th order Hermite functions formed with respect to hypercubes of side length $h$ ). Thus, $S \subset H^{1}(R)$ for $l$ any nonnegative integer. Thus, by Theorem 4.3 and (5.1), we obtain $\|u-\phi\|_{1, R} \leqq C h^{2 l-1} \sum_{\tau \in K}\left\|D^{\tau} u\right\|_{R}$.

If $u$ is in $H^{k}(R)$, let $S$ be the restriction of $k$ th order splines to $R$. Since $R$ is a bounded domain, $S$ is a finite-dimensional subspace of $H^{1}(R)$ for $k$ any positive integer. Hence, by the corollary to Theorem 4.4 and (5.1), we obtain $\|u-\phi\|_{1, R} \leqq$ $C h^{k-1}\|u\|_{k}$.

Making use of a method essentially due to J. Nitsche [6], we can find a bound for $\|u-\phi\|_{R}$ by using bounds we have found for $\|u-\phi\|_{1, R}$.

If $\phi$ is the solution to the Rayleigh-Ritz-Galerkin problem and $u$ is the generalized solution of (*), we denote $u-\phi$ as $e$. Hence, $a(e, w)=0$ for all $w$ in $S$. Let $P$ be the projection operator from $H^{1}(R)$ onto $S$, then for any $y$ in $H^{1}(R), w=P y=$ $y-(I-P) y$ for some $w \in S$ where $I$ is the identity operator on $H^{1}(R)$. Since $a(e, w)=0$, we have $a(e, y)=a(e, y-P y)$ for all $y$ in $H^{1}(R)$. Now, $e$ is in $H^{1}(R)$, so we can solve

$$
a(y, v)=\int_{R} e(x) \overline{v(x)} d x \text { for all } v \text { in } H^{1}(R)
$$

and obtain $y$ in $H^{1}(R)$.

Hence

$$
\|e\|_{R}^{2}=\int_{R} e(x) \overline{e(x)} d x=a(y, e)=a(y-P y, e) .
$$

Using the Schwartz inequality on a $((I-P) y, e)$ we obtain

$$
\|e\|_{R}^{2} \leqq C\|(I-P) y\|_{1, R}\|e\|_{1, R} .
$$

Now, if the domain $R$ is such that the solution $u$ of $a(u, v)=\int_{R} f \bar{v}$ is in $H^{2}(R)$, then by choosing $S$ to be one of the subspaces defined previously, we have $\|(I-P) y\|_{1, R} \leqq C h\|y\|_{2, R}$. If, in addition, the solution of $a(u, v)=\int_{R} f \bar{v}$ satisfies $\|u\|_{2, R} \leqq C\|f\|_{R}$, we obtain $\|(I-P) y\|_{1, R} \leqq C h\|e\|_{R}$. Hence, under the above conditions, $\|e\|_{R} \leqq C h\|e\|_{1, R}$

Following Agmon [1, p. 128], we define an open set $R$ to be of class $C^{k}$ for $k \geqq 1$ if

(1) for every $x \in \partial R$ there is an open neighborhood $U$ such that, for some $i, U \cap \partial R$ has the representation $x_{i}=g\left(x^{\prime}\right)$ for $x^{\prime}=\left(x_{1}, \cdots, x_{i-1}, x_{i+1}, \cdots, x_{N}\right)$ in $U^{\prime}$ where $U^{\prime}$ is the projection of $U$ on the hyperplane $x_{i}=0$ and $g$ is in $C^{k}\left(U^{\prime}\right)$; and if

(2) $U \cap R$ is contained in the half-cylinder $\left\{x \mid x_{i}>g\left(x^{\prime}\right)\right.$ for $\left.x^{\prime} \in U^{\prime}\right\}$.

Now, if $R$ is bounded and of class $C^{2}$, then the solution $u$ of $a(u, v)=\int_{R} f \bar{v}$ for all $v$ in $H^{1}(R)$ is in $H^{2}(R)$ and $\|u\|_{2, R} \leqq C\|f\|_{R}$ if $f$ is in $L_{2}(R)$. This is proved by Lions [5, p. 111].

Let $u$ be the solution of $a(u, v)=\int_{R} f \bar{v}$ for all $v$ in $H^{1}(R)$ and let $\phi$ in $S$ be the solution of $a(\phi, w)=\int_{R} f \bar{w}$ for all $w \in S$. Assume that $R$ is of class $C^{2}$. Then, using the splines of order 2 restricted to $R$ as $S$, we have

$$
\|u-\phi\|_{i, R} \leqq C h^{2-i}\|u\|_{2, R} \text { for } j=0,1 .
$$

If we use the Hermite subspace $H^{(1)}(R)$ as $S$, we have

$$
\|u-\phi\|_{i, R} \leqq C h^{2-i} \sum_{\tau \in K}\left\|D^{\tau} u\right\|_{R} \quad \text { for } j=0,1,
$$

where $K$ is the set of all indices $\tau$ of length 2 such that $x^{\tau}$ is not its own first Hermite interpolate. 
Furthermore, if $u$ is in $H^{l}(R)$ using the splines of order $l$ restricted to $R$ as $S$, we obtain

$$
\|u-\phi\|_{i, R} \leqq C h^{l-j}\|u\|_{l, R} \quad \text { for } j=0,1 .
$$

If $u$ is in $H^{2 m}(R)$, then using the Hermite subspace $H^{(m)}(R)$ as $S$, we obtain

$$
\|u-\phi\|_{i, R} \leqq C h^{2 m-i} \sum_{\tau \in K}\left\|D^{\tau} u\right\|_{R} \text { for } j=0,1 \text {. }
$$

We can summarize the results of this section in the following theorem and corollary.

THEOREM 5.1. If $u$, the solution of $(*)$, is in the Sobolev space $H^{k}(R)$, then there are subspaces $S_{k, h}(R)$ such that

$$
\|u-\phi\|_{1, R} \leqq C h^{k-1}\|u\|_{k, R},
$$

where $C$ is a constant independent of $h$, and $\phi$ is the solution to the Galerkin problem corresponding to (*), using $S_{k, h}(R)$ as the subspace of $H^{1}(R)$. Furthermore, if the boundary of $R$ is $C^{2}$ and $k$ is greater than or equal to 2 , then

$$
\|u-\phi\|_{i, R} \leqq C h^{k-i}\|u\|_{k, R} \quad \text { for } j=0,1 .
$$

COROLlaRY. If $f$, the right-hand side of $(*)$, is in $L_{2}(R)$ and if the boundary of $R$ is $C^{2}$, then using the splines of order 2 restricted to $R$ as the Galerkin subspace, we have

$$
\|u-\phi\|_{i, R} \leqq C h^{2-i}\|u\|_{2, R} \quad \text { for } j=0,1 .
$$

If we use the Hermite splines of order 1 restricted to $R\left(H^{(1)}(R)\right)$ as the Galerkin subspace, we obtain

$$
\|u-\phi\|_{i, R} \leqq C_{1} h^{2-i} \sum_{\tau \in K}\left\|D^{\tau} u\right\|_{R} \quad \text { for } j=0,1 .
$$

Both $C$ and $C_{1}$ are constants independent of $h$.

Finally, we remark that the subspaces constructed in Section IV can be applied in the same manner to problems of elliptic operators of arbitrary order with natural boundary conditions. They may also be used to solve Dirichlet problems using the method of least squares due to Bramble and Schatz (see [4]).

Mathematics Department

Ithaca College

Ithaca, New York 14850

1. S. Agmon, Lectures on Elliptic Boundary Value Problems, Van Nostrand Math. Studies, no. 2, Van Nostrand, Princeton, N. J., 1965. MR 31, \#2504.

2. J. Bramble \& S. Hilbert, "Estimation of linear functionals on Sobolev spaces with application to Fourier transforms and spline interpolation," SIAM J. Numer. Anal., v. 7, 1970, pp. 112-124. MR 41 \#7819.

3. J. Bramble \& S. Hilbert, "Bounds for a class of linear functionals with applications to Hermite interpolation," Numer. Math., v. 16, 1971, pp. 362-369.

4. J. BRAMble \& A. Schatz, "Least squares methods for $2 m$ th order eliptic boundaryvalue problems," Math. Comp., v. 25, 1971, pp. 1-32.

5. J. L. Lions, Problèmes aux limites dans les équations aux dérivées partielles, 2ième éd., Séminaire de Mathématiques Supérieures, no. 1 (Été, 1962), Les Presses de l'Université de Montréal, Montreal, Que., 1965. MR 40 \#4602.

6. J. Nitsche, "Ein Kriterium für die Quasi-Optimalität des Ritzschen Verfahrens," Numer. Math., v. 11, 1968, pp. 346-348. MR 38 \#1823.

7. R. VARGA, "Functional analysis and approximation theory in numerical analysis," Proceedings of the regional N.S.F. Conference at Boston Univ., July 20-24, 1970. 